INPLASY

PROTOCOL

To cite: Shi et al. Bupleurum chinense herbal formula for the antituberculosis drug-induced hepatotoxicity : A systematic review and meta-analysis. Inplasy protocol 202160088. doi:

10.37766/inplasy2021.6.0088

Received: 23 June 2021

Published: 23 June 2021

Corresponding author: Zheng Shi

361000@njucm.edu.cn

Author Affiliation: Inst Literature Chinese Med, Nanjing University of ChineseMedicine.

Support: NSFC (81904322).

Review Stage at time of this submission: Preliminary searches.

Conflicts of interest: None declared.

\section{Bupleurum chinense herbal formula for the antituberculosis drug-induced hepatotoxicity : A systematic review and meta-analysis}

\author{
Shi, Z1; Yu, S2; Chen, R3; Xue, W4; Zhou, Y5; Han, J6.
}

Review question / Objective: The aim of this meta-analysis of randomized controlled trials is to evaluate the efficacy and effectiveness of BC formula for the antituberculosis druginduced hepatotoxicity.

Condition being studied: Tuberculosis (TB), as a global concern, seriously affects people's health. The WHO global tuberculosis report 2020 estimates that in 201910.0 million people fell ill with TB. Most of the first-line drugs used in the treatment of TB have potentially hepatotoxic effects. The standard short-course chemotherapy is an effective way to control TB, which includes a combination treatment of isoniazid, rifampicin, pyrazinamide, and ethambutol. Anti-TB chemotherapy often has a long course of treatment, and the combined use of multiple anti-TB medications has a heavier burden on the liver, which easily leads to patients being forced to stop treatment. Antituberculosis drug hepatotoxicity (ATDH), which makes it difficult for patients to adhere to the entire course of chemotherapy, is the most serious adverse effect of anti-tuberculosis therapy. As an ancient therapeutic method, Bupleurum chinense(BC, Radix Bupleuri) herbal formula has been used for thousands of years in China. Since the 1990s, there have been many clinical reports of BC formula for ATDH, and the curative effect is remarkable. However, There is a lack of systematic review on the BC formula for ATDH.

INPLASY registration number: This protocol was registered with the International Platform of Registered Systematic Review and Meta-Analysis Protocols (INPLASY) on 23 June 2021 and was last updated on 23 June 2021 (registration number INPLASY202160088).

\section{INTRODUCTION}

Review question / Objective: The aim of this meta-analysis of randomized controlled trials is to evaluate the efficacy and effectiveness of $B C$ formula for the antituberculosis drug-induced hepatotoxicity. 
Condition being studied: Tuberculosis (TB), as a global concern, seriously affects people's health. The WHO global tuberculosis report 2020 estimates that in 201910.0 million people fell ill with TB. Most of the first-line drugs used in the treatment of TB have potentially hepatotoxic effects. The standard shortcourse chemotherapy is an effective way to control TB, which includes a combination treatment of isoniazid, rifampicin, pyrazinamide, and ethambutol. Anti-TB chemotherapy often has a long course of treatment, and the combined use of multiple anti-TB medications has a heavier burden on the liver, which easily leads to patients being forced to stop treatment. Antituberculosis drug hepatotoxicity (ATDH), which makes it difficult for patients to adhere to the entire course of chemotherapy, is the most serious adverse effect of anti-tuberculosis therapy. As an ancient therapeutic method, Bupleurum chinense(BC, Radix Bupleuri) herbal formula has been used for thousands of years in China. Since the 1990s, there have been many clinical reports of BC formula for ATDH, and the curative effect is remarkable. However, There is a lack of systematic review on the BC formula for ATDH.

\section{METHODS}

Search strategy: We will search PubMed, Cochrane Library, Embase, Chinese National Knowledge Infrastructure (CNKI), Wan fang Data-bases from their inception to June 22, 2021. The search strategy is: (Antitubercular Agents OR "Antibiotics, Antitubercular" OR Tuberculosis) AND (Chemical and Drug Induced Liver Injury) AND (Bupleurum). The electronic database search will be supplemented by a manual search of the reference lists of included articles.

Participant or population: Patients with liver injury after taking antituberculosis drugs. Gender and age are not limited.

Intervention: Patients were intervened with Bupleurum chinense (BC, Radix Bupleuri) herbal formula for intervention.
Comparator: Patients were intervened by other ways except BC formula. (e.g. silybin, reduced glutathione) Or stop using antituberculosis drugs, do not implement liver protection treatment.

Study designs to be included: Randomized controlled trials (RCTs) will be included.

Eligibility criteria: (1) The included studies were randomized controlled trials (RCTs) recruiting participants with ATDH. (2) For the types of interventions, the participants of the treatment groups were given a BC formula. (3) The included studies evaluated the efficacy or effectiveness of BC formula for ATDH and had clear outcome data, such as total effectiveness rate or alanine aminotransferase (ALT).

Information sources: PubMed, Cochrane Library, Embase, Chinese National Knowledge Infrastructure (CNKI), Wan fang Data-bases, Chinese Science and Technology Periodical Database(VIP) and Chinese Biomedical Database (CBM).

Main outcome(s): The effects of BC formula of ATDH were measured by the the total effective rate, ALT (alanine aminotransferase), AST (aspartate aminotransferase) and TBil (total bilirubin).

Quality assessment / Risk of bias analysis: Two reviewers will independently use the risk of bias assessment tool from the Cochrane Handbook to assess risk of bias for each publication. A third reviewer will resolves any disagreement about risk of bias. The following 7 aspects will be assessed: (1) random sequence generation; (2) allocation concealment; (3) patient blinding; (4) assessor blinding; (5) incomplete outcome data; (6) selective reporting; and (7) other sources of bias. The evaluation results were given a ranking of low risk, unclear risk, or high risk according to the above criteria.

Strategy of data synthesis: We will utilize Review Manager (Version 5.3, The Cochrane Collaboration, 2014) for data synthesis and analysis. For continuous outcomes, we will analyse studies using 
mean difference (MD) with a $95 \%$ confidence interval. MD will be calculated by subtracting after from before measurements, and standard deviation (SD) for change will be estimated by the given SD values before and after treatment. In addition, for dichotomous data, we will combine studies using a risk ratio to compare intervention and control groups with a $95 \%$ confidence interval. As the outcomes of this meta-analysis, AST, ALT, and TBil will be presented as MD, while a total effectiveness rate was presented as RR. Due to significant clinical heterogeneity, we will use a random-effects model for pooling. Heterogeneity will be evaluated statistically using the Cochran $\mathbf{Q}$ statistic and the 12 index. We will use funnel plots to evaluate the potential publication bias.

Subgroup analysis: Subgroup analysis will be performed the following different clinical heterogeneity: (1) Antituberculosis drugs were stopped after liver injury; (2) Antituberculosis drugs were not stopped after liver injury.

Sensitivity analysis: Sensitivity analysis will be performed to investigate the robustness of the pooling results by removing one trial at each turn. A $P$ value $<0.05$ will be considered statistically significant.

Country(ies) involved: China.

Keywords: Bupleurum; Antitubercular Agents; Chemical and Drug Induced Liver Injury; randomized controlled trial; Metaanalysis.

Contributions of each author:

Author 1 - Zheng Shi.

Email: 361000@njucm.edu.cn

Author 2 - Shun Yu.

Email: yushun6336@163.com

Author 3 - Renshou Chen.

Email: njcrs@126.com

Author 4 - Wenxuan Xue.

Email: 799133290@qq.com

Author 5 - Yiqun Zhou.

Email: zhouyiquntoboy@163.com
Author 6 - Jiang Han.

Email: 175478765@qq.com 